\title{
Remote Sensing and Landscapes in Sahelian Africa
}

\author{
Amélie Robert ${ }^{1}$, Marius Yao $^{2}$, Jean Louis Yengué ${ }^{3}$ and Sylvie Servain ${ }^{4}$ \\ 1. CITERES Research Laboratory, CNRS/University of Tours, Tours 37000, France \\ 2. National School of Geographic Sciences (ENSG), Marne-la-Vallée 77000, France \\ 3. Department of Geography, University of Tours, CITERES Research Laboratory, Tours 37000, France \\ 4. Department of Nature and Landscape School, National Institute of Applied Sciences (INSA Centre Val de Loire), CITERES \\ Research Laboratory, Blois 41000, France
}

\begin{abstract}
For decades, Africa has undergone many crisis, affecting economy, climate, food, politics as well as society with irreparable consequences on the environment. The protection of the latter, being one of the cornerstones of sustainable development, is only possible if it's based on a reliable and rigorous diagnosis and inventory. This study suggests a method to characterize natural resources, in particular agricultural ones, by showing their landscape context. In this perspective and in the absence of any pre-existing mapping, as it is often the case in Africa, this work provides a simple and reproducible approach that merely uses Landsat Thematic Mapper (TM) (free) images, with the only constraint of the cross-checking of several images at different times of the plant cycle.
\end{abstract}

Key words: Remote sensing, landscape, Sahelian Africa, Landsat, unsupervised classification.

\section{Introduction}

For decades, Africa was going through many crises. Economic, climate, politics and society were affected at the same time [1-4] and all of these crisis caused irreparable impacts on the environment. The fight against these attacks became synonymous with survival for the continent. This work is part of the Biosol research project, which aims at promoting new and innovative eco-agricultural knowledge and practises. Coming in the framework of ecological intensification, the latter can provide reliable answers to ensure environmental and socio-economic development in different regions of Africa. It fulfills the worldwide purpose to "feed the planet" in a sustainable way and to reduce poverty contrasts, with the use of improved endogenous and self-centered agricultural techniques. This Biosol research project also aims at responding to the numerous regional and local challenges to climate change in the

Corresponding author: Amélie Robert, Ph.D., research fields: geography, specialized in the knowledge of landscapes and their dynamics.
Sudano-Sahelian area (desertification, soil fertility loss, poverty, food shortages, forced migration...). The first results of this research project clearly show that, in the African context, the solutions are only relevant if they are based on a diagnosis and a statement of reliable and rigorous assessment of the situation [5]. But, as it's often the case in Africa, there is a lack of any pre-existing cartography. No map was available to characterize natural resources, for instance agricultural and associated landscapes in the studied areas. Knowing that this lack is a common problem for the researchers in Africa, the authors would like to automatically map the land use to find a method which can be used by other interested researchers. To draw a map from raw data is often time-consuming. The purpose of this paper is thus to suggest a method to easily and quickly map the landscapes, to know the great units, where crops are inserted. Satellite images prove to be currently the most appropriate way to know these landscapes. They are accessible to all dates (from 1972) and often freely available. Moreover, they require a much shorter processing 
time than aerial photographs.

\section{Materials and Methods}

\subsection{Materials}

The chosen area is at the edge of the Sahelian and Sudano-Sahelian areas. It often suffers from climate change, such as increasing violence rainfall in the rainy season, intensification and extension of the dry season [1], affecting agricultural activities and submitting the populations to the risk of hunger. Being very difficult to reach, especially in the rainy season, because of its marginal position from the main roads of the country, the rural village of Barani is almost devoid of all studies and research on its agricultural practices.

Barani is a village of nearly 4,000 inhabitants. It is located in North-Western Burkina Faso next to the border with Mali [6]. The main activity of the villagers remains agriculture composed of traditional crops (millet, fonio, sorghum, beans, ground peas, peanuts, sesame, hibiscus, corn, etc.) and extensive breeding of cattle (zebu), goats (goats and sheep) and poultries (pigeons, guinea fowls and chickens). This agriculture is heavily dependent on climatic constraints. Barani is located on the $700 \mathrm{~mm}$ isohyets, in the Sudano-Sahelian climate [7] with two seasons (a dry season and a rainy season), and the alternation depends on the swing of the Intertropical Convergence Zone (ITCZ).

In this area, the dry season lasts from October to May. It's characterized by near-zero rainfall and a warm wind that dries the area. No agriculture is then possible, all the more since irrigated crops are not a usual practise. Conversely, from June to September, the rainy season brings rains and wet winds. It's the growing season. Farmers are busy in the fields to cultivate what represents the only source of food.

To know the landscapes of this area, the satellite, from which the selected images are taken, must be chosen and several criteria must then be taken into account in this selection. One of the most important is the resolution, because it determines the identification of terrestrial objects. There are two kinds: spatial resolution and spectral resolution. The first one sets the pixel size, that is the smallest unit of the image, so the size of the smallest identifiable object. The spectral resolution determines the number of bands, from which information can be drawn, and as a consequence, the greater or lesser ease of identification of objects. The choice of the satellite is based on the objective of the research conducted and the characteristics of the considered area. It should also take into account the characteristics of the objects the authors want to identify.

As part of this work, the authors study the main categories of land use (crops, spontaneous vegetation and bare soil), focusing in particular on cultivated fields. Moreover, they know that the area of these fields in the studied sector is between 0.38 ha and 26 ha, thanks to a field survey [8]. Consequently, the sensor should provide high spectral and spatial resolutions. Far from being the only one, Landsat 5 Thematic Mapper (TM) could correspond, because its spatial resolution is $30 \mathrm{~m}$ $\times 30 \mathrm{~m}$ and it measures the luminance in seven bands, the visible (blue, green, red), thermal infrared, near infrared (NIR) and mid-infrared (MIR), where the two last ones inform on the structure and the water status of the vegetation, respectively, whereas the green one indicates the chlorophyllian activity.

Furthermore, the images offered by this sensor are free downloadable on United States Geological Survey (USGS) website available for the chosen dates, and cover an area wide enough to encompass the studied area; the track of the satellite Landsat $5 \mathrm{TM}$ is $185 \mathrm{~km}$ wide. Landsat 7 ETM+, launched more recently (in 1999), offers the same advantages and thus could have been selected, but the failure of one of its correctors in May 2003 prompted us to reject it.

In order to get the best discrimination between the three main classes of land use, the choice of the selected dates proves to be essential; it is based on empirical knowledge of the field. In June, cultivated 
lands were recently planted and they are thus more easily distinguished from grasslands. Nevertheless, they can be confused on the images with soils, which are bare or covered by a scattered vegetation. An image taken in June should thus be selected. September is considered as a supplementary favorable month. Indeed, at this time of year, chlorophyllian activity is strong. Moreover, cultures are ready to be harvested and they may then be confused with grasslands, but they can be distinguished from bare soils.

Considering images that were taken at these two periods, removes any ambiguity between cultures and other land use categories. Devineau and Serpantié [9], whose research also aimed to identify the Burkinabe landscapes by remote sensing, advocated to add a third image, taken in January. In this study, the purpose is not a fine discrimination of different plant formations, but the identification of the main categories of land use, that is why this additional image does not seem necessary. Two images were acquired in order to draw up an inventory of existing landscapes. They respectively date from 24th of June and 7th of October 2009.

\subsection{Methods}

The method consists in several nested processings that are summarized in Fig. 1.

\subsubsection{Creating New Bands}

The processing of the two acquired images was realized by using Envi software that is adapted for geospatial analysis and spectral image processing. The first work consisted in processing raw bands into new bands. The purpose was to reduce the number of data by selecting the most relevant. Indeed, the bands (seven for Landsat TM) of the same scene are often correlated with one another, resulting in a repetition of the information. Thus, the data can be summarized. These new bands are then created by processing. They are indices that synthetize the information. One of

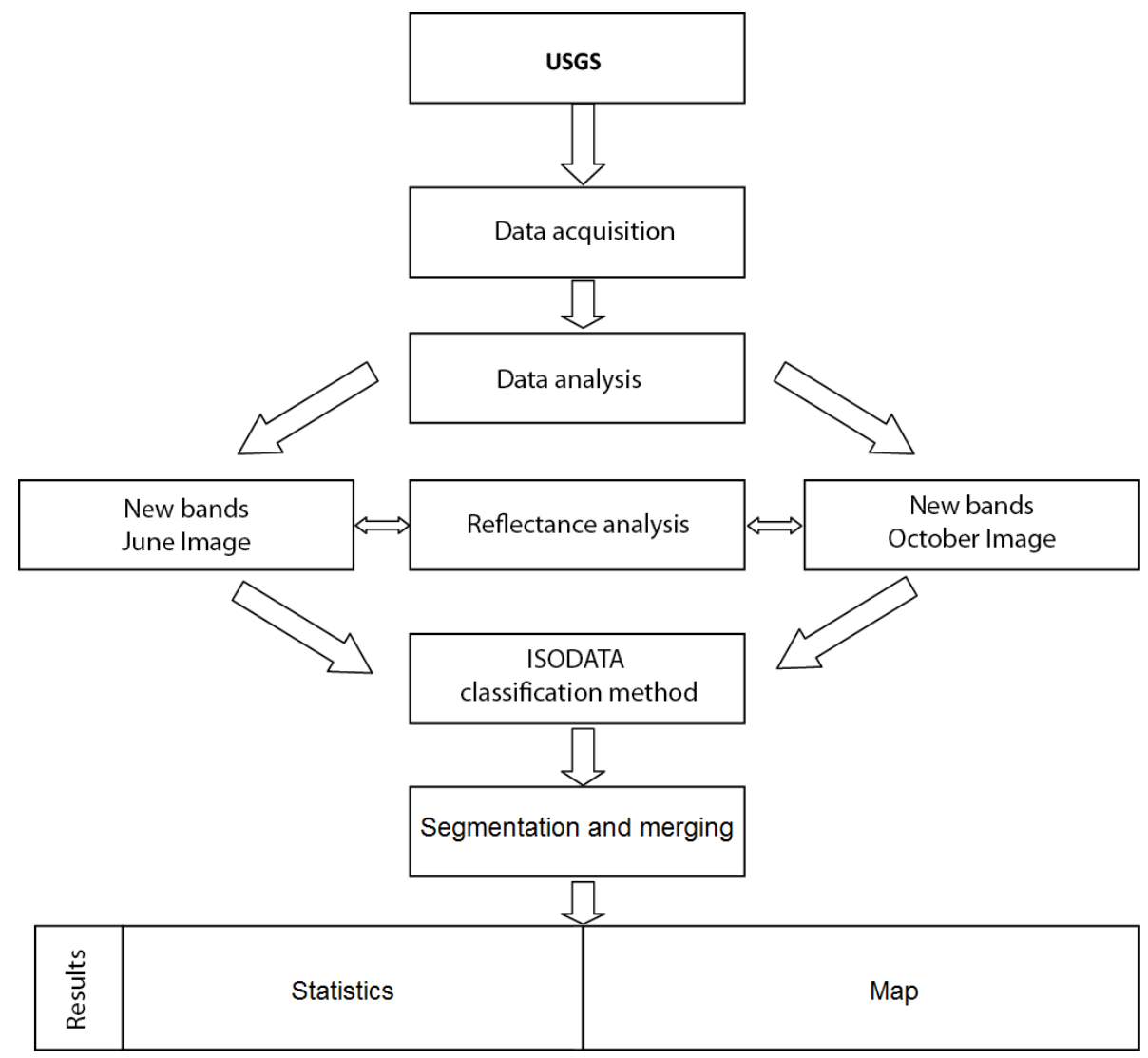

Fig. 1 Methodology implemented for the land use mapping. 
these indices is the normalized difference vegetation index (NDVI) that shows the vegetation state, the plant growing stage. The data recorded by the satellite are converted into information, from which the characteristics of the landscape units can be learnt, such as biomass, leaf cover, soil moisture, etc..

Two new bands were considered. Their choice was supported by pre-existing works, in particular those conducted by Devineau and Serpantié [9]:

(1) NDVI, which highlights the chlorophyllian vegetation and its variations;

(2) Index of brightness (IB), which highlights the bare soil.

The required information is thus synthesized in two created bands, instead of the original seven ones, for each selected image. These new bands were used for the creation of a color composite.

\subsubsection{Color Composite}

This tool allows an easier analysis of the synthesized information. A color composite is generated by the software, using three primary colors (red, green and blue). Only one band or neo-band should be associated to each of these colors. Thus, only three (neo-) bands can be used in this processing. Red, green and blue colors vary from 0 to 255, according to the values taken by each pixel in the three chosen (neo-) bands. Consequently, these three colors are combined in various proportions and the pixels appear in different colors on the obtained image that results from the association of the three colors/of the three (neo-) bands. This obtained image is a digital image called color composite.

In this study, the chosen (neo-) bands are the new bands, which the authors created in the last stage, in other words IB and NDVI, knowing that one band is lacking (Fig. 2); the IB of October, considered as the least relevant, must be rejected, because only three bands can be considered in a color composite. In the end of the processing that is realized by the software, the authors obtained a color composite (Fig. 3).

\subsubsection{Classification}

The color composite is a digital image. It synthetizes the information, but because the purpose of the paper was to realize a land use map, each pixel of this image should then be classified and associated with a land use category. The Envi software was used to offer different kind of classifications supervised or not. This work aimed at defining a method that would be quickly and easily reproducible, that is why the authors opted for an unsupervised classification method. This one is based on Isodata technique-Iterative Self-Organizing Data Analysis that was proposed by Ball and Hall [10].

The interest of this classification is that it does not require a prior knowledge of the land use, even if this knowledge can not be ignored for the validation of the classification. The classification is thus more based on the spectral signatures of the objects than on exogenous information. Every pixel of the image is automatically sorted by the software according to its

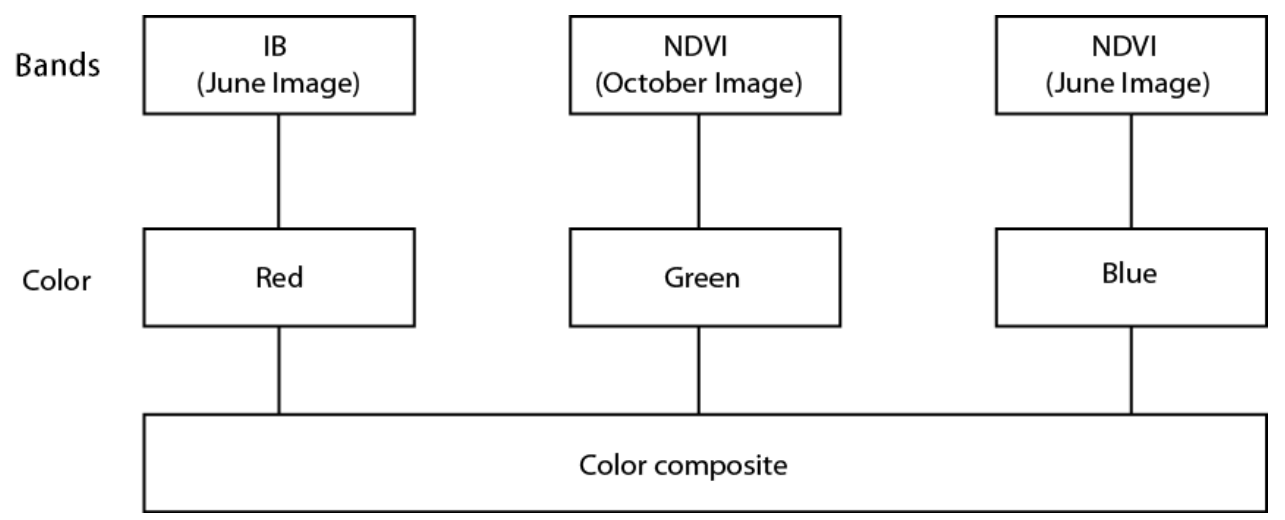

Fig. 2 Color composite. 


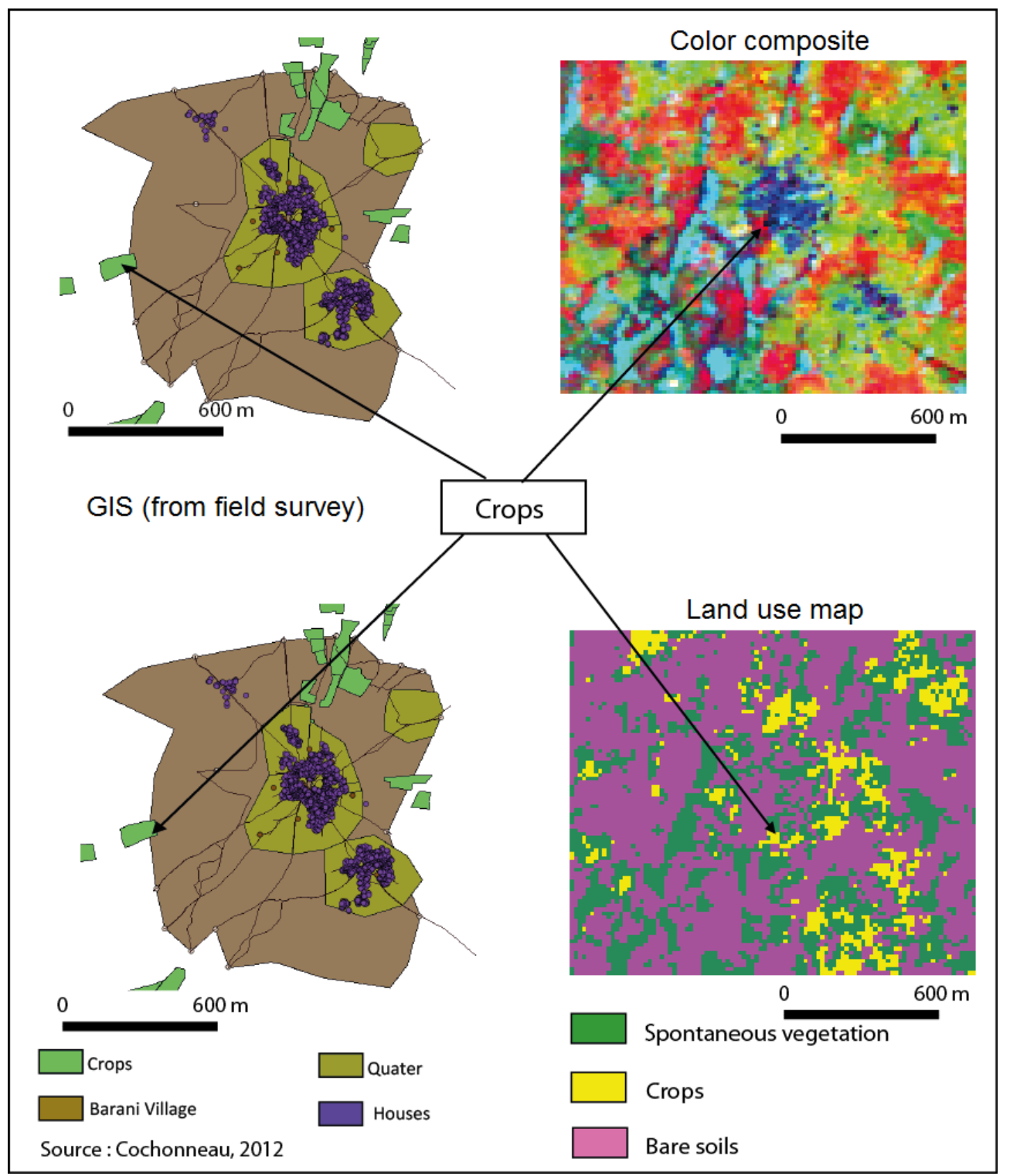

Fig. 3 Confrontation of the obtained results for the identification of crops.

The color composite (red = June IB, green = October NDVI, blue = June NDVI) in Fig. 2 and the classification (land use map) are compared with the GIS [8] for the identification of crops.

values taken in the chosen bands. The principle of Isodata classification is as follows: the pixels are associated with the class, whose centers are closest to the values that they take in these chosen bands; the process is repeated, and at each iteration, these cluster centers are recalculated.

In this work, the chosen bands are the ones which the authors selected for the color composite (Fig. 2). Other variables must be predefined to process an Isodata classification. The software requires that the minimum and maximum numbers of classes and the minimum number of pixels per class desired are specified, like the class grouping threshold and the maximum number of iterations. These variables are not known a priori, even the number of classes. Indeed, this one, which the authors obtained in the end of the processing, has often to be greater than the number of finally expected classes, because within the classes, the spectral differences can be larger. To get the most relevant result, which is closest to the ground reality, different combinations of these variables have thus to be tested. 


\section{Results}

\subsection{Color Composite}

The obtained color composite (Figs. 3 and 4) associates the red color to IB of June, the green color to the October NDVI and the blue color to the June NDVI (Fig. 2). Whereas, croplands appear in yellow and bare soils in red, spontaneous vegetation (in this case, savannahs) appears according to their characteristics, in colors ranging from blue-green to dark green, including cyan.

The relevance of the new bands-IB and NDVI, and the selected dates - 24th of June and 7th of
October 2009, can be perceived in this correspondence. Indeed, each main land use category is well distinguished. The highest values taken by bare soil are in the IB, explaining that they appear in red on the color composite; the highest values taken by the savannahs are in the NDVI of June and/or October, explaining also their colors on the color composite in the same proportions - the mix of blue and green giving the cyan color (Fig. 4). As for the croplands, they take their highest values in the June IB and October NDVI, and they are thus in yellow on the color composite - the mix of red and green giving the yellow color (Fig. 3).

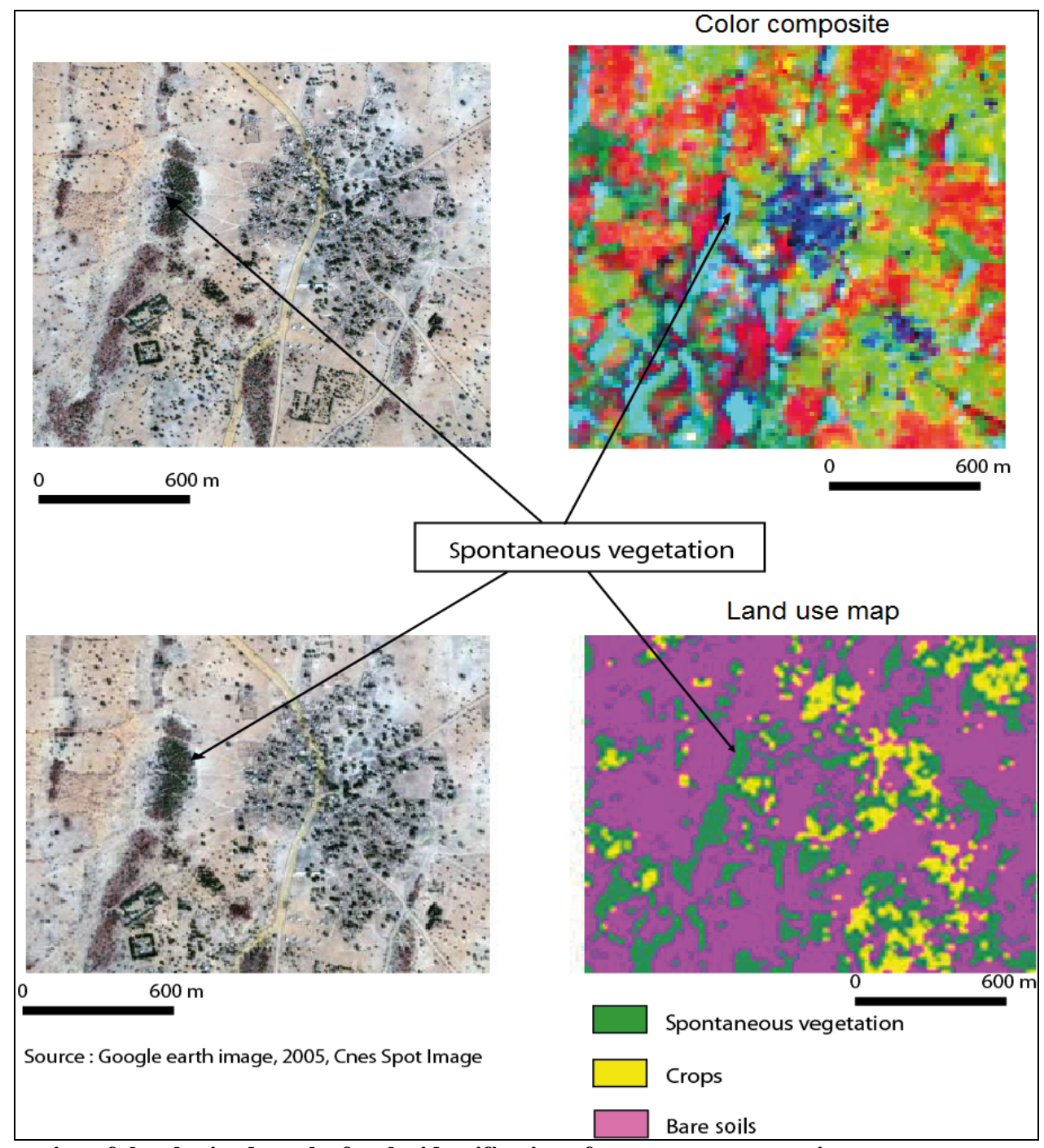

Fig. 4 Confrontation of the obtained results for the identification of spontaneous vegetation.

The color composite (red = June IB, green = October NDVI, blue = June NDVI) in Fig. 2 and the classification (land use map) are compared with the Google earth image for the identification of spontaneous vegetation. 
Consider only one date would cause mix-up with one class or the other one. Indeed, in June, the cultivated lands are more or less bare (which can explain the strong values taken in the June IB), and in October, ready to be harvested, crops mix up with spontaneous vegetation (hence the strong values also recorded in October NDVI).

Visual analysis of the colored composite is facilitated by prior knowledge of the field and is supported by an analysis of the spectral signatures of detected objects (values taken by each object in the different unchanged bands). The colored composite is a necessary tool. But, it provides only intermediate results in a process, leading to the completion of a land use map; that is why the authors associated it with an automatic classification.

\subsection{From the Classification to the Map of Land Use}

Unsupervised classifications, based on Isodata method, were performed and tested. The spectral signatures of obtained classes were analyzed. The results were also compared to the color composite and data from the field survey (Figs. 3 and 4). In the end of this work, the classification providing the best matches was selected. It was obtained by entering the following variables ahead of the process performed by the software: a range of classes between three and five, and three iterations.

The result of this automatic process was a classification, in which five classes were distinguished. They were then identified and associated with the tallying categories of land use, thanks to the authors' prior knowledge of the field and the analysis of the spectral signatures of objects included in each class. The classes that were associated with the same land use category were merged and this led to the map of land use of Barani in 2009, where only the three main land use categories are distinguished (Fig. 5).

This map shows the extent of bare soil and the scattering of the cultivated land on the selected area. Some of the latter are concentrated around the two residential areas, the main village and its hamlet in the South. Others are away from them. Two landscape units can be distinguished:

(1) In the North of the village, bare soils alternate with savannahs. In some areas, the vegetation can not grow and this can be explained by the outcrops of breastplate;

(2) In the South, the organization is less strict, less geometric; savannahs extend further, especially in the Southeast, not in lines like in the North, but in the form of oil stains.

\section{Discussion}

To suggest relevant solutions in the framework of this research project, the authors needed a diagnosis and a statement of reliable and rigorous assessment of the situation [5] in the studied area. But, the authors were facing with the absence of any pre-existing mapping. Their objective was to find a simple and reproducible method to map the landscapes, to know the great units where crops are inserted. Satellite images prove to be currently the most appropriate source, especially because they require a much shorter processing time than aerial photographs. The authors selected Landsat TM images; one reason is that they are freely downloadable on USGS website. The authors' work demonstrates that they are also suitable for the characterization of plant resources in dry Africa. Many other authors [11-13] systematically add images taken by other sensors, e.g., Satellite for Earth Observation (SPOT), but this increases the financial and temporal costs. The method, which the authors suggested, only uses Landsat TM images with one constraint - the cross-checking of two images taken at different times of the plant cycle. To identify also the Burkinabe landscapes by remote sensing, Devineau and Serpantié [9] add a third image taken in January. But, with regard to the authors' purpose, i.e., knowing the great landscape units, this one appeared not necessary. As the authors demonstrated it, two images taken at two times of the plant cycle are enough to 


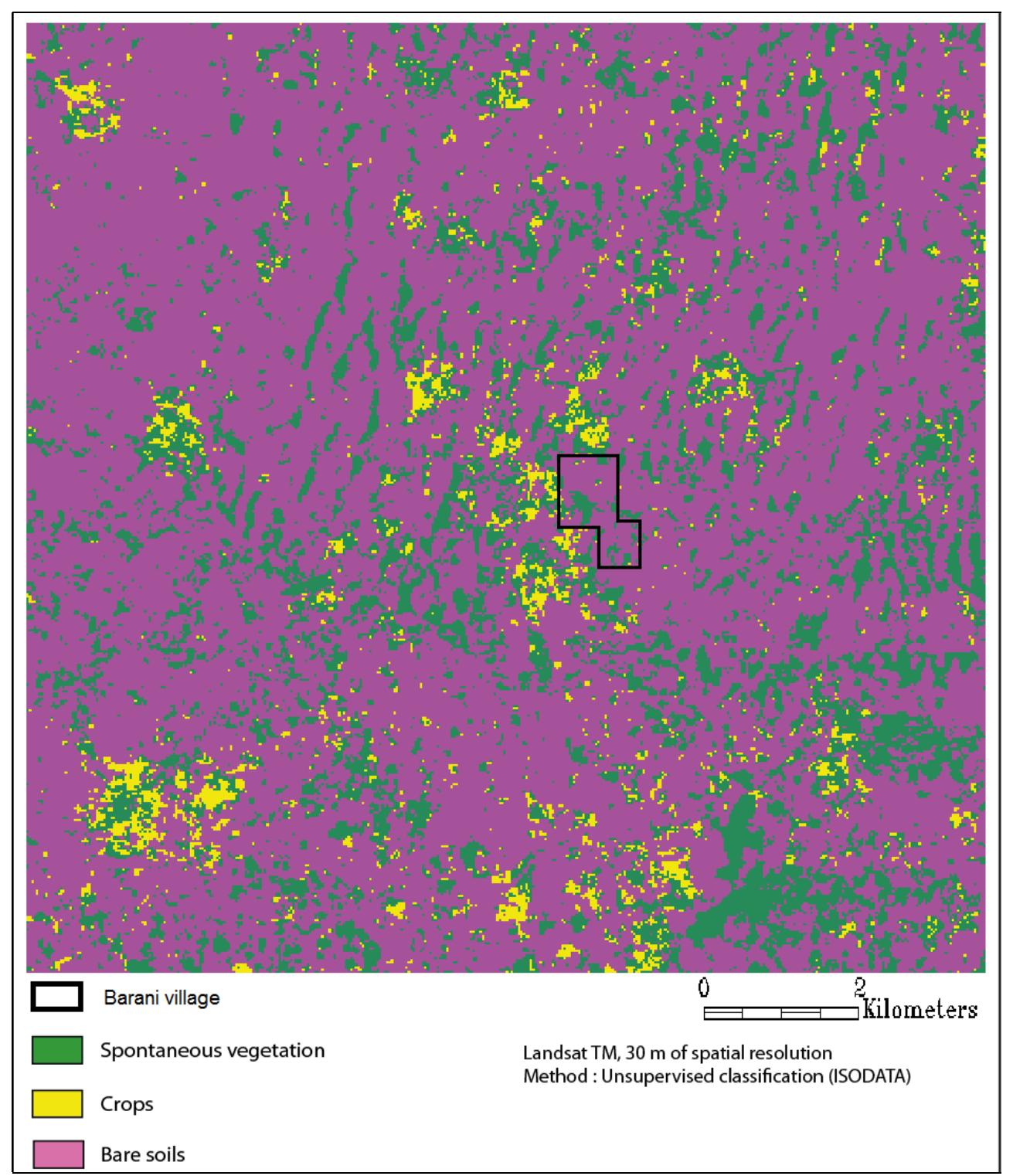

Fig. 5 Map of land use of Barani in 2009.

distinguish the main land use categories to avoid all mix-ups. The authors' methodology is the result of extensive testing. It's validated by the confrontation with other sources, especially with field surveys (Figs. 3 and 4). Indeed, it was experienced in an area where the authors knew perfectly to ensure its efficiency. In the end of the process, thanks to the obtained map, they are able to describe the great landscape units, where crops are inserted.

\section{Conclusions}

Basing on extensive testing, this work leads to the characterization of the great landscape units in the studied Sahelian area. It was essential, because the first result of the authors' research showed the necessity of a prior diagnosis and assessment of the situation in the studied area and because the authors were faced with the absence of any pre-existing mapping. This work consisted of defining a simple and reproducible method using free satellite images (Landsat TM images) with only one constraint-the cross-checking of two images taken at different times of the plant cycle. Free Landsat TM images appear suitable for the characterization of plant resources in 
dry Africa. The confrontation of the obtained map with other sources, especially with field surveys, validates the authors' method. The same processing is currently being implemented on other sites in Africa and the first results confirm its relevance.

\section{Acknowledgments}

This work is part of the Biosol research project, which was funded by the Centre-Val de Loire region in France. The authors would like to express their acknowledgement for its financial support. They also thank Burkinabé, who warmly welcomed them during their field trip.

\section{References}

[1] Bandre, E. 1993. "The Dynamic of Vegetation Cover in Gboué Area, Kossi Province, Burkina Faso.” In Aspects of Natural Environments of Burkina Faso. Talence: University of Bordeaux III, 27-46. (in French)

[2] Parmentier, B. 2007. Feeding Humanity: The Great Problems of the World Agriculture in the XXIst Century. Paris: La Découverte. (in French)

[3] Griffon, M. 2006. Feeding the World: For a Doubly Green Revolution. Paris: O. Jacob. (in French)

[4] Guillaume, F. 2009. Defeating Hunger to Be Done with What Is Unacceptable. Paris: Eyrolles. (in French)

[5] Yengué, J. L., and Cochonneau, M. 2013. "For the Development of Agroecology at Barani (Burkina Faso).”
Journal of Agricultural Science and Technology B 3 (8): 545-50.

[6] Limet, C. 2011. "GIS and International Aid: From Small Scale to Large Scale.” M.Sc. thesis, University of Orleans. (in French)

[7] Lerebours-Pigeonnière, A., and Menager, M. T. 2001. Atlas of Burkina Faso. Paris: J. A. Publisher. (in French)

[8] Cochonneau, M. 2012. "Agroecology in Barani (Burkina Faso): Decrypting an Agrarian System.” M.Sc. thesis, University of Tours. (in French)

[9] Devineau, J. L., and Serpantié, G. 1991. "Project SALT: Plant Landscapes and Agrarian Systems in Burkina.” In Characterization and Monitoring of Terrestrial Environments in Arid and Tropical Areas, edited by Pouget, M. Bondy: ORSTOM, 373-85. (in French)

[10] Ball, G. H., and Hall, D. J. 1965. ISODATA: A Novel Method of Data Analysis and Pattern Classification. Technical Report, Stanford Research Institute, Menlo Park, California, USA.

[11] Viet Bui, D., and Goïta, K. 2007. "Use of Satellite Images to Study Flood Risk Linked to the Land Use in Northern Viet Nam.” Remote Sensing Journal 7 (1-4): 203-15. (in French)

[12] Nicolas, K., Souleymane, P., Lamourdia, T., and Halidou, C. 2002. "Assessment of the Mid-infrared TM5 Band for the Identification of Crops and Natural Vegetation in the Cotton Area of Burkina Faso.” Remote Sensing 2 (3): 183-92. (in French)

[13] Lambin, E. 1998. “The Contribution of Remote Sensing to Study Agrarian Systems in Africa: Burkina Faso Example.” Journal of the International African Institute 58 (3): 337-52. (in French) 